\title{
POUR UNE PHILOSOPHIE HYBRIDÉE DE LA BIOLOGIE
}

\author{
Charles T. Wolfe
}

Association Multitudes | « Multitudes »

2004/2 $\mathrm{n}^{0} 16 \mid$ pages 11 à 14

ISSN 0292-0107

Article disponible en ligne à l'adresse :

https://www.cairn.inforevue-multitudes-2004-2-page-11.htm

Distribution électronique Cairn.info pour Association Multitudes.

(C) Association Multitudes. Tous droits réservés pour tous pays.

(cC) BY-NC 


\section{pour une}

philosophie

hybridée

dela

biologie

Charles T. Wolfe 
Le post-modernisme est obsédé par le corps et terrifié par la biologie. Le corps est un thème diablement populaire dans les "cultural studies" - mais il s'agit ici du corps plastique, reconfigurable, socialement construit, non pas de cette parcelle de matière qui tombe malade, qui pourrit et qui meurt. La créature, l'individu qui émerge de la pensée post-moderne est décentré, hédoniste, s'inventant et s'adaptant sans cesse. Il ressemble plus à un publicitaire de Los Angeles qu'à un pêcheur indonésien.

Terry Eagleton, After Theory.

L a pensée post-moderne est obsédée par le corps et terrifiée par la biologie: voilà un homme qui n'a pas froid aux yeux! N ous laisserons de côté la pensée propre d'E agleton, qui a récemment décou vert que son marxisme le menait ve rs le christianisme de son enfance, et nous retiendrons la remarque suivante: dans toute une gamme de pensées qui disent se soucier du corps, on n'a jamais constaté autant de désintérêt pour le travail scientifique qui le prend comme objet, disons la biologie, ou la réflexion sur le vivant. $\mathrm{N}$ 'oublions pas que le premier penseur de la multitude, B ento Spinoza, pour utiliser le prénom portugais qu'il affectionnait, était fort préoccupé par ce corps dont Eagleton déplore l'oubli, y compris au sens prisé par D eleuze où «nul ne sait ce que peut un corps ». U n corps est aussi composé d'autre corps plus petits, tout en composant lui-même des corps plus grands: ainsi l'être humain qui se croit souverain dansl'universest comme le «ver dans le sang »qui croit tout connaître du corps dans lequel il vit (lettre XXXII, à O Idenburg). Sans en faire un «père »de la philosophie de la biologie et surtout de ses avatars passés en revue ici, espérons qu'il aurait bien voulu lui servir de parrain.

Proposons alors deux buts pour le dossier qui suit : d'abord rendre plus difficiles de tels jugements péremptoires, sans pour autant se poser contre l'hédonisme ni chercher à replacer le pêcheur indonésien au centre d'un discours théorique qui l'au rait ignoré, mais plutôt en revenant sur la biologie comme objet de réflexion philosophique, au lieu de la laisser aux héi ti ers du positivisme logi que; ensuite, sur le plan strictement théorique, combler une lacune qui existe entre deux discours.

$D$ 'une part, la réflexion dans ce que l'on appelle «épistémologie »en F rance, discipline qui, après une glorieuse activité entre les années I920 et I950, et auréolée d'un certain prestige humain (K oyré, Cavaillès, Canguilhem), présente maintenant un certain retard, un état de veille et de contemplation de sa gloire ancienne, et surtout un certain aspect «réactif »qui fait qu'elle a tendance à opposer une vision soi-disant plus «riche »historiquement ou socialement au «réductionnisme »anglo-saxon; on le voit égal ement dans la réponse constituée par I' «École de Paris » 
autour de F rancisco Varela, récemment disparu, à l'intelligence artificielle ou plutôt au nouveau paradigme «computationnel »: réponse d'inspiration phénoménologique et particulièrement merleau-pontyenne, qui met l'accent sur ce qu'on a envie d'appeler «incarnation » (embodiment), mais que ses défenseurs préfèrent traduire par «in sc ription corporelle ». L'intérêt porté par F élix G uattari àVarela est un des rares exemples d'une pensée «post-moderne »(on sait le peu de goût que G uattari avait pour cette notion) qui s'intéresse au vivant autrement que pour y célébrer un potentiel d'artificialité, ou y fêter les noces du «genre ».

$D$ 'autre part, nous sommes coutumiers d'un discours sur le vivant né de la philosophie politique, qui travaille à partir de catégories mystérieuses pour le public intellectuel plus large, comme «bio-politique ». C elui-ci pourrait remplacer dans le jeu structuraliste des cases vides, l'ancien discours d'inspiration marxiste qui critiquait le darwinisme, le «tout génétique » ou la réduction de l'esprit au cerveau (voir notamment les travaux de Richard L ewontin, de Steven Rose ${ }^{\mathrm{I}}$ et de Steven Jay Gould). M ais il ne le fait guère, puisque le vivant en soi ne semble pas focaliser I'attention des théoriciens du bio-politique, sinon sous une forme anthropologique ou métaphorique; c'est toujours du vivant en tant qu'objet de contrôle ou sujet de domination. II a en quelque sorte été abandonné aux praticiens de la «bio-éthique », discipline qui sert surtout à faire renouveler par sa présence des budgets de recherche dans différents laboratoires américains ou européens. C e dossier va donc tenter de réintégrer une réflexion conceptuelle sur la biologie au sein du questionnement politique qui est le nôtre, comme on le voit par exemple dans l'entretien avec John Symons.

L'exemple du darwinisme est toujours vivace, dans la réflexion sur le vivant où les «armes »n' ont jamais cessé de parler. Pendant qu'il continue d'être considéré avec méfiance de ce côté de l'Atlantique - en souvenir des remarques de $M$ arx sur l'influence de $M$ althus dans la formation de la pensée de $D$ arwin, sans doute - il est devenu le paradigme incontournable, majoré de génétique, pour toute réflexion, non seulement biologique, mais appartenant à la philosophie de la biologie, outre-Atlantique. II est curieux de noter à ce propos que l'utilitariste contemporain et défenseur de l'eugénisme, Peter Singer, a récemment publié un petit livre au titre éloquent, $A$ Darwinian Left: I'objectif étant de «reprendre » D arwin à la droite qui en avait fait sa chasse gardée... On retrouve la «vieille gauche » mais sous une nouvelle forme, car l'adversaire a repris à son compte l'argumentaire post-moderne qui met sur le même plan tous les «discours »ou «récits», avec la conséquence que la droite religieuse dure en Amérique insiste sur le fait que le récit 
de la $G$ enèse a donc une valeur égale au paradigme de la sélection nat urelle, et à ce titre doit être enseigné au lycée; ce thème a surtout été étudié en $\mathrm{F}$ rance par $\mathrm{D}$ ominique L ecourt, par exemple dans L'Amérique entre la Bible et Darwin. Enfin, sur le plan de la stricte inventivité scientifique, des personnages aussi différents que G erald E delman et JeanJacques $\mathrm{K}$ upiec (dans ce dossier) retrouvent des phénomènes darwiniens à des niveaux insoupçonnés de la matière vivante, et d'une autre manière, plus notionnelle, $M$ athieu Aury tente ici une lecture del'œuvre du philosophe américain contemporain $D$ aniel $D$ ennett axée sur son aspect darwinien, pour construire encore un pont, encore une hybridation, vers une vision politique d'inspiration foucaldienne.

Plutôt que de «ponts » ou de «liens »toujours faciles à défaire, peutêtre vaut-il mieux en effet décrire ce dossier comme une galerie de portraits ( $d$ ')hybrides On y trouvera à la fois des réflexions d'ensemble sur l'état des lieux de cette discipline aux contours flous, la philosophie de la biologie, considérée en tant que discipline universitaire constituée (dans mon entretien avec John Symons ainsi que dans l'étude de T imo K aitaro sur l'ouvrage de F rançois D uchesneau, Philosophie de la biologie); des «études de cas » délimitées historiquement qui cherchent à mettre en lumière certains «grands problèmes » (la notion d'organisme dans mon article, celle de vitalisme dansl'article d'Alexandre M étraux) ; enfin, des travaux contemporains qui cherchent, dans l'espace limité imparti ici, à montrer quelques-unes des «pratiques »intellectuelles existant sous ce vocable général de philosophie de la biologie (la nouvelle lecture philosophique de D arwin chez D ennett, examinée par Mathieu Aury; une philosophie du corps dans son artificialité foncière, proposée par Bernard Andrieu), ainsi que la présentation par un biologiste, Jean-Jacques K upiec, de sa notion d'un «darwinisme au niveau cellulaire », notion qui entre en résonance de manière multiple avec plusieurs autres contri butions, tant sur le plan métaphysique (qu'est-ce qu'un élément? qu'est-ce qu'un monde? où se situe le gène, l'organisme ou l'individu embryonniaire là-dedans?) que sur le plan moral (si la théorie de la sélection naturelle est «vraie», suis-je encore libre?).

$\mathrm{N}$ ous ne cherchons donc pas ici à construire un «dialogue » entre traditions rivales, type même de tentative qui est périmée très vite, et condamnée de plus à rester parasitaire sur ces traditions, mais plutôt à élargir modestement l' «assiette », le «tour de table » des réflexions sur la philosophie de la biologie aujourd'hui.

(I) N otamment Nous ne sommes pas programmés : génétique, hérédité, idéologie, par Richard L ewontin, Steven Rose et L eon K amin, Paris, La D écouverte, 1985, et plus récemment, de Rose, Lifelines. Biology, Freedom, Determinism, L ondres, Penguin, 1997. 